\title{
Evaluation of ocular surface glycocalyx using lectin-conjugated fluorescein
}

This article was published in the following Dove Press journal:

Clinical Ophthalmology

13 August 2010

Number of times this article has been viewed

\section{Hiroshi Mochizuki' \\ Masaki Fukui ${ }^{1,2}$ \\ Shin Hatou ${ }^{1,2}$ \\ Masakazu Yamada' \\ Kazuo Tsubota ${ }^{2}$}

'Division for Vision Research, National Institute of Sensory Organs, National Tokyo Medical Center, Tokyo, Japan; ${ }^{2}$ Department of Ophthalmology, Keio University School of Medicine, Tokyo, Japan
Correspondence: Masakazu Yamada Division for Vision Research, National Institute of Sensory Organs, National Tokyo Medical Center, 2-5-I Higashigaoka, Meguro-ku, Tokyo I52-8902, Japan

Tel +8I 334 II 0 III ext 5236

$\mathrm{Fax}+8 \mid 334110185$

Email yamadamasakazu@kankakuki.go.jp
Purpose: A glycocalyx plays important roles in the ocular surface epithelium, but there is no direct simple method to evaluate ocular surface glycocalyx. We tested a wheat germ agglutinin conjugate of fluorescein (F-WGA) as a marker to demonstrate ocular surface glycocalyx in vivo.

Methods: After a 5\% F-WGA solution was applied to the eyes of eight healthy volunteers, fluorescent intensities of the central cornea and bulbar conjunctiva were measured by fluorophotometry. A $10 \%$ fluorescein-conjugated dextran solution served as the control. Changes in fluorescent intensities of the ocular surface following a challenge with $5 \% \mathrm{~N}$-acetyl cysteine, a conventional mucolytic agent, were tested in the second experiment. Saline instillation served as a control.

Results: The ocular surface was diffusely stained by F-WGA. Breakup of the precorneal tear film was not apparent, possibly because F-WGA was bound to the glycocalyx of the ocular surface epithelium. F-WGA fluorescent intensities were high in the superior, nasal, and inferior regions of the bulbar conjunctiva and low in the temporal conjunctiva and cornea. No such regional differences were observed with fluorescein-conjugated dextran. F-WGA fluorescent intensities decreased significantly with $\mathrm{N}$-acetyl cysteine instillation, whereas they were not affected by saline instillation.

Conclusion: The fluorophotometric method may be used to evaluate the glycocalyx quantitatively in the ocular surface in vivo.

Keywords: cornea, fluorescein, glycocalyx, lectin, mucin

\section{Introduction}

A glycocalyx, which consists of mucopolysaccharides and glycoproteins, is found on the apical portion of microvilli in corneal and conjunctival epithelia. ${ }^{1}$ Membraneassociated mucins produced by apical cells are major components of the glycocalyx of the ocular surface epithelium. ${ }^{1-4}$ A glycocalyx plays important roles in ocular surface lubrication by maintaining wettability and eliminating foreign bodies and pathogens. ${ }^{1,5,6}$ Thus, the roles of glycocalyx-including mucins in the ocular surface have attracted attention. ${ }^{1-7}$

Several methods have been developed to evaluate ocular surface mucins. Rose bengal and lissamine green have been used clinically to determine epithelial damage of the conjunctiva. Rose bengal stains ocular surface epithelial cells when they are not covered with mucins, ${ }^{7,8}$ whereas lissamine green stains membrane-damaged epithelial cells. ${ }^{9}$ Impression cytology is another technique that has been used for the analysis of ocular surface mucins. The density of goblet cells, mRNA expression of 
mucin genes, and alterations in glycosylation characteristics of mucins have been analyzed using this technique. ${ }^{10-13}$ Although recent advances in ocular surface mucins research are significant, the development of a simple and quantitative method to evaluate ocular surface glycocalyx will enable an improved understanding of the role of glycocalyx including mucins in the pathogenesis of ocular surface disorders.

In the present study, we tested a lectin conjugate of fluorescein as a marker to demonstrate the presence of ocular surface glycocalyx in vivo. Lectins are a group of glycoproteins that bind specifically with carbohydrate residues of glycoconjugates. ${ }^{14}$ Of these, wheat germ agglutinin (WGA) specifically binds to $\mathrm{N}$-acetyl glucosamine and $\mathrm{N}$-acetyl neuraminic acid. ${ }^{14} \mathrm{~A}$ number of histochemical studies have demonstrated WGA binding to the ocular surface glycocalyx. ${ }^{15-20}$ In light microscopic studies, WGA has high affinity for apical cells of the corneal and conjunctival epithelium and for goblet cells in the conjunctiva. ${ }^{15-18}$ WGA binding to the glycocalyx of ocular surface cells has been confirmed by transmission electron microscopy. ${ }^{19,20}$ Based on these experimental studies, ${ }^{14-20}$ we used a WGA conjugate of fluorescein (F-WGA) to evaluate the glycocalyx in the ocular surface in vivo.

\section{Subjects and methods Subjects}

Eight healthy volunteers (five males and three females) aged $27-46$ years $(33.4 \pm 6.8$ years, mean \pm standard deviation [SD]) with no history of eye disease, except for refractive errors, were enrolled in the study. At the screening visit, one of the authors (MF) performed routine ocular examination of all subjects, followed by an examination of the ocular surface, including Schirmer testing and measurement of tear film breakup time. Saline solution $(2 \mu \mathrm{L})$ containing $1 \%$ fluorescein was used for vital staining. All subjects had more than $5 \mathrm{~mm}$ of Schirmer strip wetting, more than five seconds in tear film breakup, and no apparent fluorescein staining of the cornea and conjunctiva. All subjects were diagnosed as normal according to the Japanese criteria for dry eye syndrome. ${ }^{21}$ The following experiments of F-WGA were done on subsequent investigation visits.

The guidelines of the World Medical Association Declaration of Helsinki were followed. The subjects received a full explanation of the procedures and provided their informed consent for participation prior to the experiment. The protocol was approved by the institutional review board of National Tokyo Medical Center (R-07-011), and all subjects provided written informed consent.

\section{Fluorescent dye and fluorophotometer}

F-WGA (molecular weight 38,000 Da) and fluoresceinconjugated dextran (molecular weight 40,000 Da) were purchased from Molecular Probes Inc. (Eugene, OR). Both dyes have their longest wavelength absorption maximum at $494 \mathrm{~nm}$ and an emission spectrum that peaks at $518 \mathrm{~nm}$. A $10 \%$ F-WGA stock solution and a 10\% fluoresceinconjugated dextran solution were prepared in sterile $0.067 \mathrm{M}$ phosphate-buffered saline, $\mathrm{pH}$ 7.4.

A commercial slit-lamp fluorophotometer (Anterior Fluorometer FL-500, Kowa Co. Ltd., Tokyo, Japan) was used to quantify fluorescent intensity. The illuminating light was focused as a $2 \mathrm{~mm}$ diameter circle on the corneal surface. The emitted light passed through a band interference filter centered on $565 \mathrm{~nm}$ (half bandwidth, $25 \mathrm{~nm}$ ) and was directed to a photomultiplier tube with the band interference filter centered on a wavelength of $490 \mathrm{~nm}$ (half bandwidth, $30 \mathrm{~nm}$ ).

A $10 \%$ F-WGA solution $(100 \mathrm{mg} / \mathrm{mL}$ was diluted in phosphate-buffered saline to produce standards ranging from $0.001 \%$ to $10 \%$ for calibration. A $10 \%$ fluorescein-conjugated dextran solution was diluted to produce standards ranging from $0.001 \%$ to $10 \%$ in the same manner. A cuvette was constructed by gluing together two microscopic slides and two cover glasses. The cover glasses were sandwiched by the two slides to provide a $12-15 \mu \mathrm{m}$ thick space for the fluid layer. A fresh cuvette was used for each solution. The standards $(10 \mu \mathrm{L})$ were added to the cuvettes, and fluorescence intensity was measured by slit-lamp fluorophotometer. The interaction of F-WGA or fluorescein-conjugated dextran with proteins was also tested by diluting the standards in phosphatebuffered saline containing $10 \%$ fetal bovine serum.

\section{Staining characteristics of F-WGA}

Preliminary experiments were conducted to determine the optimal concentration and time to observe F-WGA staining using four eyes from two subjects. Different concentrations of F-WGA solutions ranging from $1 \%$ to $10 \%$ were tested. A slit-lamp fluorophotometer was used to quantify the fluorescent staining of the ocular surface. The instrument was focused on the central cornea and nasal bulbar conjunctiva $3 \mathrm{~mm}$ posterior from the limbus, and the background fluorescent intensity at each point was measured. A volume of $5 \mu \mathrm{L} \mathrm{F-WGA} \mathrm{solution} \mathrm{was} \mathrm{applied} \mathrm{to} \mathrm{the} \mathrm{eye} \mathrm{using} \mathrm{an}$ Eppendorf micropipette. The fluorescent intensities of the central cornea and nasal bulbar conjunctiva were measured for 30 minutes (every minute for the initial five minutes, and every five minutes from 5-30 minutes). Fluorescent staining 
pattern was observed using a slit-lamp biomicroscope equipped with a blue-free barrier filter (SL-D7, Topcon Corp., Tokyo, Japan). ${ }^{22}$

\section{Differences in fluorescent intensities by ocular surface regions}

A slit-lamp fluorophotometer was used to quantify the fluorescent staining of the ocular surface. The subjects were seated in front of the fluorophotometer. The instrument was focused on the central cornea and eight quadrants (superior, superonasal, nasal, inferonasal, inferior, inferotemporal, temporal, and superotemporal) of the bulbar conjunctiva $3 \mathrm{~mm}$ posterior to the limbus, and the background fluorescent intensity at each point was measured. A 5\% F-WGA solution $(5 \mu \mathrm{L})$ was applied to the right eye using an Eppendorf micropipette. Five minutes later, the fluorescent intensities of the central cornea and the eight quadrants of the bulbar conjunctiva were measured. The same procedure was performed after application of $5 \mu \mathrm{L} 10 \%$ fluorescein-conjugated dextran solution to the left eye.

\section{Alteration of F-WGA staining by applying $\mathrm{N}$-acetyl cysteine}

The effect of N-acetyl cysteine, a conventionally used mucolytic agent, on F-WGA staining was tested. ${ }^{23}$ A 5\% N-acetyl cysteine (Sigma-Aldrich, St Louis, MO) solution was prepared in sterile $0.067 \mathrm{M}$ phosphate-buffered saline, $\mathrm{pH} 7.4$.

The background fluorescent intensities were measured at the central cornea and four quadrants (superior, nasal, inferior, and temporal) of the bulbar conjunctiva $3 \mathrm{~mm}$ posterior from the limbus. A volume of $5 \mu \mathrm{L}$ of $5 \% \mathrm{~F}-\mathrm{WGA}$ solution was applied to both eyes using an Eppendorf micropipette. Five minutes later, the fluorescent intensities of the central cornea and four quadrants of the bulbar conjunctiva were measured. A drop $(20 \mu \mathrm{L})$ of $5 \% \mathrm{~N}$-acetyl cysteine was instilled into the right eye using an Eppendorf micropipette. A drop $(20 \mu \mathrm{L})$ of saline $(0.9 \% \mathrm{NaCl}$ solution $)$ was instilled into the left eye in the same manner. The abovementioned procedure was repeated three times at two-minute intervals. The fluorescent intensities of the central cornea and the four quadrants of the bulbar conjunctiva were then measured to identify the effects of $\mathrm{N}$-acetyl cysteine challenge.

\section{Statistical methods}

Data were analyzed using InStat 3 software (GraphPad Software, Inc., La Jolla, CA). The results are given as mean $\pm \mathrm{SD}$ ). The significance of the differences was analyzed with Kruskal-Wallis test or Wilcoxon matched pairs test. A $P$ value $<0.05$ was considered statistically significant.

\section{Results}

\section{Fluorescent dye and fluorophotometer}

We tested the safety profile of F-WGA using rabbit eyes, and then human eyes, although this material is not considered hazardous according to its material safety data sheet. No signs of inflammation or damage were detected either immediately or 24 hours after instilling five drops of $10 \%$ F-WGA solution at 10-minute intervals into the eyes of four rabbits. Instillation of one drop of $10 \% \mathrm{~F}-\mathrm{WGA}$ solution into the eyes of four healthy subjects caused no discomfort, and no adverse effects were detected by a slit-lamp examination. From these preliminary experiments, a F-WGA solution below $10 \%$ was considered to have no apparent adverse effects on the ocular surface.

Fluorescent intensities of the standard dye solutions in cuvettes were measured by slit-lamp fluorophotometer. The relationship between fluorescent intensity and F-WGA concentration was linear $\left(r^{2}=0.986\right)$, and the data generated by this method were consistent and reproducible. Fluorescent intensity was unaffected by the presence of $10 \%$ fetal bovine serum. Similar results were obtained when the fluoresceinconjugated dextran standards were tested $\left(r^{2}=0.978\right)$.

\section{Staining characteristics of F-WGA}

Results of preliminary experiments using four eyes of two subjects to determine the optimal concentration and time to observe F-WGA staining are shown in Figures 1 and 2, respectively. The fluorescent intensities at the central cornea and nasal bulbar conjunctiva increased as the concentrations of F-WGA solution increased from $1 \%$ to $3 \%$. However, they reached a plateau with F-WGA concentrations between $3 \%$ and $10 \%$. The fluorescent intensities at the central cornea and the nasal bulbar conjunctiva did not decay with time, and remained stable for 30 minutes after application of a $5 \%$ F-WGA solution. From these preliminary experiments, we selected a 5\% F-WGA solution and the fluorophotometric measurement was performed five minutes after the application. A 10\% fluorescein-conjugated dextran solution was selected because it provided fluorescent intensities similar to that obtained on application of a 5\% F-WGA solution to the eye.

The F-WGA staining patterns of the ocular surface observed using a slit-lamp biomicroscope equipped with a blue-free barrier filter are shown in Figure 3. F-WGA stained the corneal surface with a faint diffuse pattern. There was 


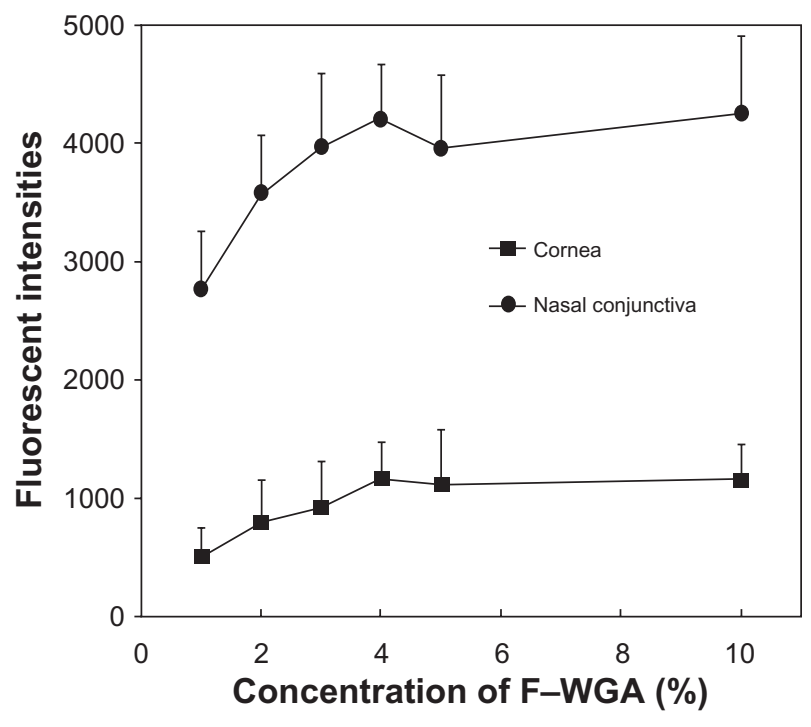

Figure I Plot of fluorescent intensities and wheat germ agglutinin conjugate of fluorescein (F-WGA) concentration. The fluorescent intensities at the central cornea and nasal bulbar conjunctiva increased as the concentrations of F-WGA solution increased from $1 \%$ to $3 \%$. They reached a plateau with F-WGA concentrations between $3 \%$ and $10 \%$.

no apparent break-up of the precorneal tear film as observed with fluorescein sodium, possibly because F-WGA was mainly bound to the corneal surface. Staining of the bulbar conjunctiva was more intense than the cornea, which had a diffuse but somewhat granular pattern. Tear mucus was strongly stained with F-WGA. These staining patterns were stable for at least 60 minutes, although the fluorescence was not detectable on the next day.

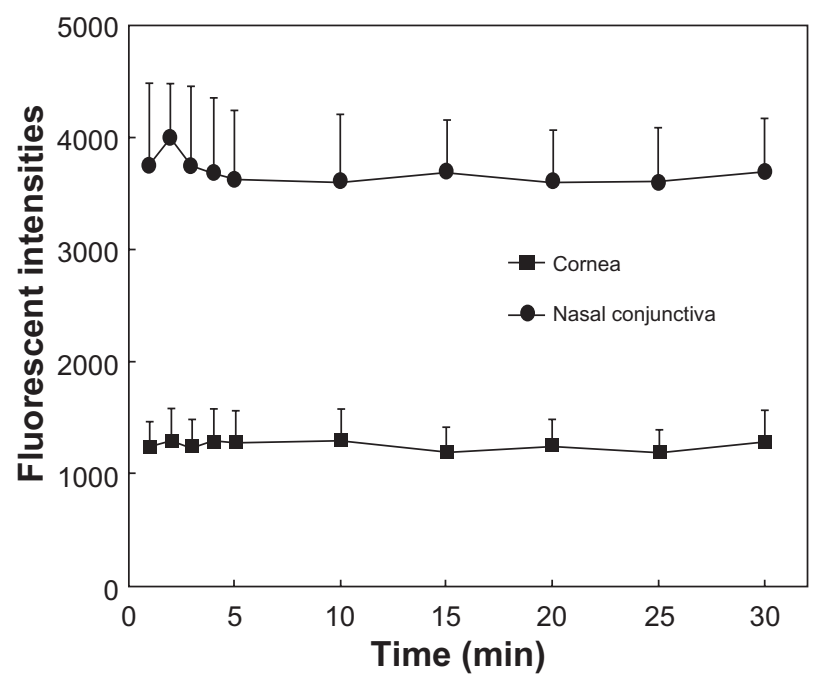

Figure 2 Plot of fluorescent intensities and time after application of $5 \%$ wheat germ agglutinin conjugate of fluorescein (F-WGA) solution. The fluorescent intensities of the central cornea and nasal bulbar conjunctiva were measured for 30 minutes. The fluorescent intensities at the central cornea and the nasal bulbar conjunctiva did not decay with time, and remained stable for 30 minutes after application of a $5 \%$ F-WGA solution.
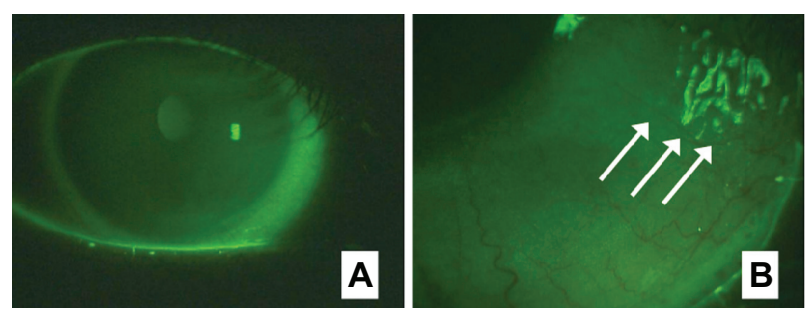

Figure 3 Staining patterns of wheat germ agglutinin conjugate of fluorescein (F-WGA) on the ocular surface observed using a slit-lamp biomicroscope equipped with a blue-free barrier filter. A) The corneal surface was stained by F-WGA with a faint, diffuse pattern. Staining of the bulbar conjunctiva was more intense than the cornea. B) Tear mucus was strongly stained with F-WGA (arrows).

\section{Differences in fluorescent intensities by ocular surface regions}

Fluorescent intensities five minutes after the applications of F-WGA and fluorescein-conjugated dextran are shown in Figures 4 and 5, respectively. Both dyes showed higher fluorescent intensities at the bulbar conjunctiva than at the central cornea. When fluorescein-conjugated dextran was applied, there were no differences in the fluorescent intensities of the bulbar conjunctiva regions. In contrast, F-WGA fluorescent intensities were high in the superior, nasal, and inferior regions of the bulbar conjunctiva, and low in the temporal region. Fluorescent intensities of F-WGA in the inferotemporal, temporal, and superotemporal regions were significantly lower than those in the superior region $(P<0.05$, Kruskal-Wallis test $)$.

\section{Alteration of F-WGA staining by applying $\mathrm{N}$-acetyl cysteine}

Changes in fluorescent intensities by challenging with $\mathrm{N}$-acetyl cysteine or saline are shown in Figures 6 and 7,

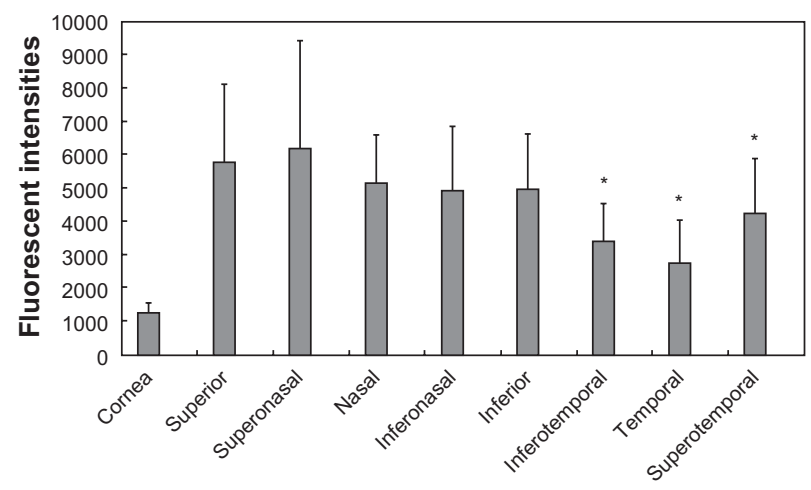

Figure 4 Fluorescent intensities at the central cornea and eight quadrants of the bulbar conjunctiva after application of the wheat germ agglutinin conjugate of fluorescein (F-WGA). F-WGA showed higher fluorescent intensities at the bulbar conjunctiva than at the central cornea. F-WGA fluorescent intensities in the inferotemporal, temporal, and superotemporal regions of the bulbar conjunctiva were significantly lower than those in the superior region ( $P<0.05$, Kruskal-Wallis test). 


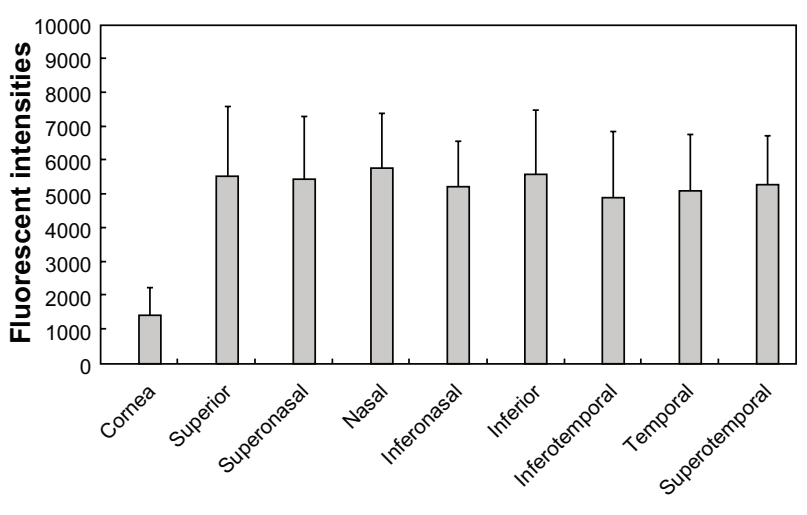

Figure 5 Fluorescent intensities at the central cornea and eight quadrants of the bulbar conjunctiva after application of fluorescein-conjugated dextran. There were no regional differences in the conjunctiva with fluorescein-conjugated dextran.

respectively. The decrease in F-WGA fluorescent intensities at the cornea and the bulbar conjunctiva was statistically significant $(P<0.01$ at the cornea and the nasal and inferior regions of the bulbar conjunctiva and $P<0.05$ at the superior and temporal regions of the bulbar conjunctiva; Wilcoxon matched pairs test). F-WGA fluorescent intensities were not affected by instillation of saline.

\section{Discussion}

In the present study, we attempted to evaluate the ocular surface glycocalyx in vivo using F-WGA. Lectins, glycoproteins derived from plants and animals, have the unique ability to bind to specific glycoconjugate carbohydrates. ${ }^{14}$ WGA is reported to bind to several specific carbohydrates,

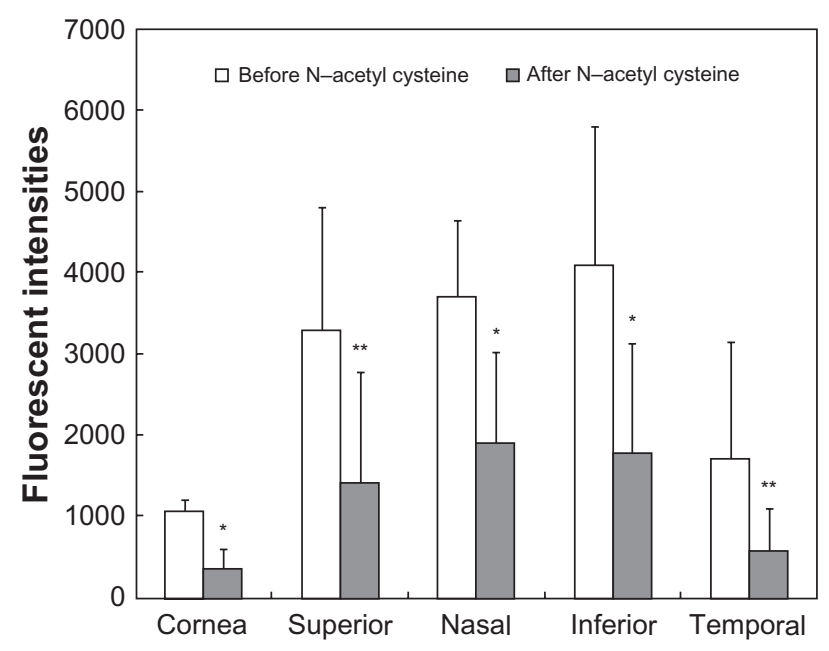

Figure 6 Fluorescent intensities of the wheat germ agglutinin conjugate of fluorescein (F-WGA) at the central cornea and four quadrants of the bulbar conjunctiva before and after a challenge with $\mathrm{N}$-acetyl cysteine. The decrease in $\mathrm{F}$-WGA fluorescent intensities at the cornea and the bulbar conjunctiva was statistically significant $(* P<0.01$ at the cornea and the nasal and inferior regions of the bulbar conjunctiva and $* * P<0.05$ at the superior and temporal regions of the bulbar conjunctiva; Wilcoxon matched pairs test).

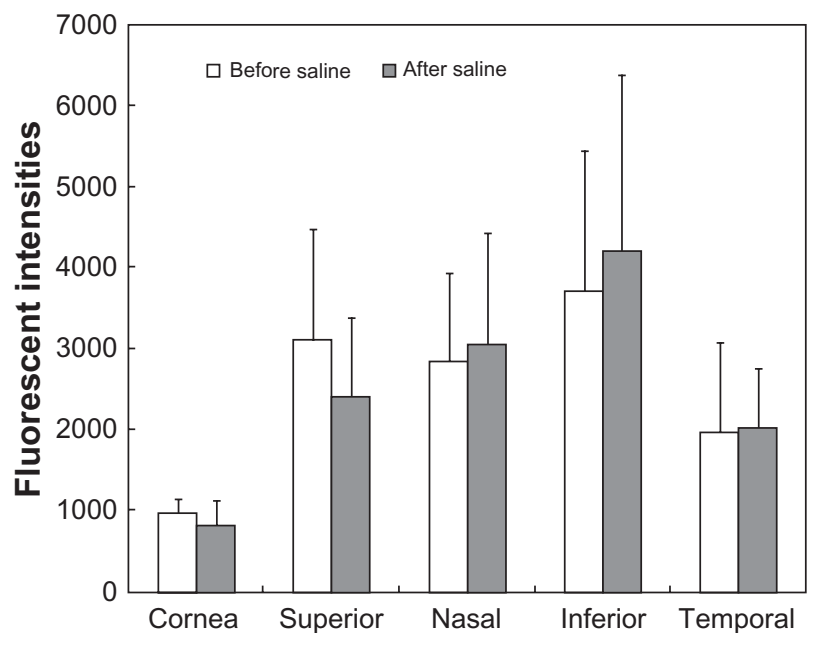

Figure 7 Fluorescent intensities of the wheat germ agglutinin conjugate of fluorescein (F-WGA) at the central cornea and four quadrants of the bulbar conjunctiva before and after a saline challenge. F-WGA fluorescent intensities were not affected by saline instillation.

such as $\mathrm{N}$-acetyl glucosamine and $\mathrm{N}$-acetyl neuraminic acid. ${ }^{14}$ No apparent break-up of the precorneal tear film appeared after application of the F-WGA solution. The F-WGA fluorescent intensities at the central cornea and the nasal bulbar conjunctiva did not decay with time, at least for 30 minutes. Based on published histochemical studies that have investigated binding of lectins to the ocular surface, ${ }^{15-20} \mathrm{~F}-\mathrm{WGA}$ appears to bind predominantly to the glycocalyx of the ocular surface epithelium.

$\mathrm{N}$-acetyl cysteine is a classic mucolytic agent, which depolymerizes the mucin glycoprotein oligomers by hydrolyzing the disulfide bonds that link the mucin monomers. ${ }^{23}$ Berry et al have recovered various types of mucins, including MUC1, MUC2, MUC4, and MUC5AC, from the ocular surface by washing with $\mathrm{N}$-acetyl cysteine. ${ }^{23}$ In our study, F-WGA fluorescent intensities decreased dramatically after instilling $\mathrm{N}$-acetyl cysteine, whereas they were not affected by saline instillation. As a result of the $\mathrm{N}$-acetyl cysteine challenge, some parts of mucins, which are the major components of the glycocalyx, are removed from the ocular surface as shown by Berry et al. ${ }^{23}$ These results appear to provide additional evidence suggesting that F-WGA binds to ocular surface glycocalyx.

F-WGA fluorescent intensities were higher in the superior, nasal, and inferior regions of the bulbar conjunctiva than in the temporal region. We used fluorescein-conjugated dextran, the molecular weight of which is similar to that of F-WGA. There were no differences in the fluorescent intensities in the bulbar conjunctiva regions when the fluoresceinconjugated dextran was applied. Therefore, it is unlikely that the region-by-region differences in the barrier function of 
conjunctival epithelium are responsible for the difference in fluorescent intensities. Although the reason for the phenomenon is not clear, some researchers have reported that there are topographic differences in the distribution of conjunctival goblet cells. ${ }^{24,25}$ Goblet cells densities are higher in the nasal conjunctiva than in the temporal region and higher in the palpebral conjunctiva than in the bulbar conjunctiva. ${ }^{24,25}$ Light microscopy studies have shown that WGA has a high affinity for goblet cells in the conjunctiva. ${ }^{15,17}$ Topographic differences in F-WGA fluorescent intensities may reflect these topographic distributions in conjunctival goblet cells.

In conclusion, we evaluated ocular surface mucins using F-WGA. This fluorophotometric method appears to be promising and may enable us to evaluate ocular surface mucins quantitatively in vivo. The data presented were obtained from healthy adults. The clinical significance of F-WGA staining should be tested in subjects with various ocular surface disorders, such as dry eye, allergic conjunctivitis, and contact lens wear. Further investigations have been planned to clarify these issues.

\section{Acknowledgments}

This study was supported in part by a grant from the Ministry of Health, Labor, and Welfare, Japan and by a grant from the Ministry of Education, Culture, Sports, Science, and Technology, Japan.

\section{Disclosure}

The authors have no proprietary interest in any materials used in this work.

\section{References}

1. Gipson IK, Argüeso P. Role of mucins in the function of the corneal and conjunctival epithelia. Int Rev Cytol. 2003;231:1-49.

2. Inatomi T, Spurr-Michaud S, Tisdale AS, Gipson IK. Human corneal and conjunctival epithelia express MUC1 mucin. Invest Ophthalmol Vis Sci. 1995;36:1818-1827.

3. Pflugfelder SC, Liu Z, Monroy D, et al. Detection of sialomucin complex (MUC4) in human ocular surface epithelium and tear fluid. Invest Ophthalmol Vis Sci. 2000;41:1316-1326.

4. Argüeso P, Spurr-Michaud S, Russo CL, Tisdale A, Gipson IK. MUC16 mucin is expressed by the human ocular surface epithelia and carries the H185 carbohydrate epitope. Invest Ophthalmol Vis Sci. 2003; 44:2487-2495.

\section{Clinical Ophthalmology}

\section{Publish your work in this journal}

Clinical Ophthalmology is an international, peer-reviewed journal covering all subspecialties within ophthalmology. Key topics include: Optometry; Visual science; Pharmacology and drug therapy in eye diseases; Basic Sciences; Primary and Secondary eye care; Patient Safety and Quality of Care Improvements. This journal is indexed on Submit your manuscript here: http://www.dovepress.com/clinical-ophthalmology-journal
5. Nagyová B, Tiffany JM. Components responsible for the surface tension of human tears. Curr Eye Res. 1999;19:4-11.

6. Tiffany JM. The normal tear film. Dev Ophthalmol. 2008;41:1-20.

7. Feenstra RP, Tseng SC. What is actually stained by rose bengal? Arch Ophthalmol. 1992;110:984-993.

8. Feenstra RP, Tseng SC. Comparison of fluorescein and rose bengal staining. Ophthalmology. 1992;99:605-617.

9. Chodosh J, Dix RD, Howell RC, Stroop WG, Tseng SC. Staining characteristics and antiviral activity of sulforhodamine B and lissamine green B. Invest Ophthalmol Vis Sci. 1994;35:1046-1058.

10. Nelson JD, Havener VR, Cameron JD. Cellulose acetate impressions of the ocular surface. Dry eye states. Arch Ophthalmol. 1983;101: 1869-1872.

11. Danjo Y, Watanabe H, Tisdale AS, et al. Alteration of mucin in human conjunctival epithelia in dry eye. Invest Ophthalmol Vis Sci. 1998;39: 2602-2609.

12. Singh R, Joseph A, Umapathy T, Tint NL, Dua HS. Impression cytology of the ocular surface. Br J Ophthalmol. 2005;89:1655-1659.

13. Hori Y, Argüeso P, Spurr-Michaud S, Gipson IK. Mucins and contact lens wear. Cornea. 2006;25:176-181.

14. Wright CS. Structural comparison of the two distinct sugar binding sites in wheat germ agglutinin isolectin II. J Mol Biol. 1984;178:91-104.

15. Kawano K, Uehara F, Sameshima M, Ohba N. Application of lectins for detection of goblet cell carbohydrates of the human conjunctiva. Exp Eye Res. 1984;38:439-447.

16. Holmes MJ, Mannis MJ, Lund J, Jacobs L. Lectin receptors in the human cornea. Cornea. 1985-86;4:30-34.

17. Prause JU, Jensen OA, Paschides K, Støvhase A, Vangsted P. Conjunctival cell glycoprotein pattern of healthy persons and of patients with primary Sjögren's syndrome - light microscopical investigation using lectin probes. J Autoimmun. 1989;2:495-500.

18. Rittig M, Brigel C, Lütjen-Drecoll E. Lectin-binding sites in the anterior segment of the human eye. Graefes Arch Clin Exp Ophthalmol. 1990; 228:528-532.

19. Versura P, Maltarello MC, Bonvicini F, Caramazza R, Laschi R. Detection of mucus glycoconjugates in human conjunctiva by using the lectin colloidal gold technique in TEM. A quantitative study in normal subjects. Acta Ophthalmol. 1986;64:445-450.

20. Wells PA, DeSiena-Shaw C, Rice B, Foster CS. Detection of ocular mucus in normal human conjunctiva and conjunctiva from patients with cicatricial pemphigoid using lectin probes and histochemical techniques. Exp Eye Res. 1988;46:485-497.

21. Shimazaki J, Tsubota K, Kinoshita S, et al. Definition and diagnosis of dry eye 2006. Atarashii Ganka. 2007;24:181-184. Japanese.

22. Koh S, Watanabe H, Hosohata J, et al. Diagnosing dry eye using a blue-free barrier filter. Am J Ophthalmol. 2003;136:513-519.

23. Berry M, Ellingham RB, Corfield AP. Human preocular mucins reflect changes in surface physiology. Br J Ophthalmol. 2004;88:377-383.

24. Kessing SV. Mucous gland system of the conjunctiva. A quantitative normal anatomical study. Acta Ophthalmol. 1968;95 Suppl:1-133.

25. Rivas L, Oroza MA, Perez-Esteban A, Murube-del-Castillo J. Topographical distribution of ocular surface cells by the use of impression cytology. Acta Ophthalmol. 1991;69:371-376.

\section{Dovepress}

PubMed Central and CAS, and is the official journal of The Society of Clinical Ophthalmology (SCO). The manuscript management system is completely online and includes a very quick and fair peer-review system, which is all easy to use. Visit http://www.dovepress.com/ testimonials.php to read real quotes from published authors. 